\title{
Awareness of COVID-19 among patient attendants coming to the emergency department in a tertiary care center in Hyderabad Metropolitan region: A questionnaire-based survey
}

\author{
Mulla Mahammad Rafi, Mohammed Ismail Nizami*, Uppaluri Sarath Chandra, Ashima Sharma, Gaddam \\ Dharani Reddy, Lubna Tarannum
}

Department of Emergency Medicine, Nizam's institute of medical sciences Panjagutta, Hyderabad -500082, Telangana, India

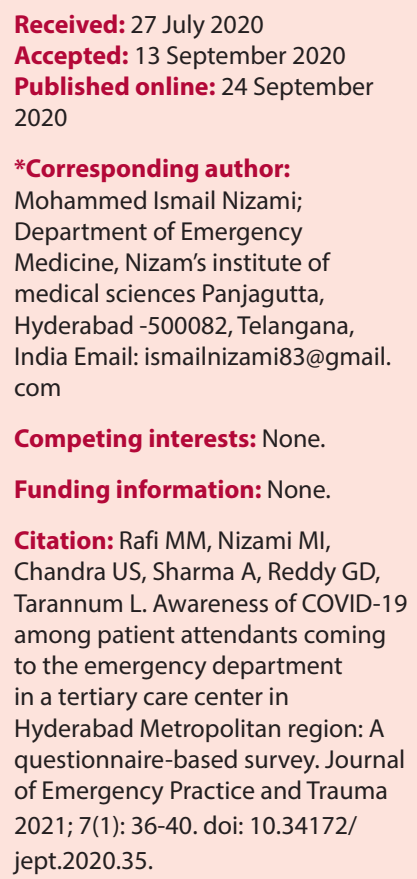

\begin{abstract}
Objective: COVID-19 has become a major public health concern in recent times with detrimental effects on health and economy of the nation exerting unbearable pressure on health care personnel. It is vital for the caretakers and the people accompanying those patients to be aware of the ongoing pandemic and its impact on the society and to realize their responsibility towards curbing the spread of the disease. The aim of this study is to assess the awareness of COVID-19 among the attendants accompanying the patients at emergency department (ED) and to determine their perception of the disease.

Methods: A questionnaire was prepared and the attendants were approached with it. The attendants responded to the questionnaire by choosing the appropriate answers. Illiterates were explained about the questions in the language they understood and the responses were recorded. The survey was conducted from May $25^{\text {th }}, 2020$ to June $10^{\text {th }}$, 2020. Descriptive analysis was done to represent the study characters.

Results: A total of 509 participants responded to the questionnaire with the mean age between 30 to 50 years. Participants were fairly conversant of the preventive measures but a significant portion lacked the knowledge of transmission and the impact of COVID-19. Education was observed to have a positive correlation with the correct responses given. Conclusion: Public education for the spread of awareness is yet to reach the illiterate section of the society. The need exists for educating the attendants of the patients as well who actually are potential candidates for contracting and also for spreading the disease. Keywords: Awareness, COVID-19, Attendants, Emergency department
\end{abstract}

\section{Introduction}

COVID-19 is an ongoing pandemic caused by the 2019 novel corona virus (2019 n-CoV) and is a major global health concern. The novel corona virus began as an outbreak of pneumonia of unknown cause in Wuhan, Hubei province of China in December 2019 (1). It was declared as Pandemic by the World Health Organization (WHO) on $11^{\text {th }}$ March, 2020 and COVID-19 has spread to more than 188 countries and territories (2).

As of today, there are over 22158645 confirmed cases and the disease has claimed more than 781366 lives globally (3). Currently, the United States leads the chart in the burden of corona followed by Brazil and India (3). India as of today has about 2767235 active cases and the count is increasing daily (3).
In Nepal, a cross-sectional web-based study was conducted by Sarraf et al, in the month of April, 2020 to assess the knowledge about universal safety precautions of general public. A total of 228 people responded to the web-based study and $57.9 \%$ of them scored less than $80 \%$ of the total knowledge score (more than $80 \%$ to be considered as excellent knowledge and less than that to be considered average knowledge), showing that the majority of study participants had average knowledge (4).

Zhong et al. conducted a cross sectional online questionnaire-based survey from January 27th to February 1st, 2020 in Hubei province of China to assess the knowledge, attitude and practices of Chinese residents towards COVID-19. The questionnaire contained questions based on clinical presentations, transmission 
and preventive aspects. The final sample included 6910 participants and the findings showed that residents who belonged to relatively high socio-economic strata and were mainly women had higher knowledge, optimistic attitude and practices towards COVID-19 (5).

\section{Methods}

A questionnaire was prepared and the survey was conducted between 25th May, 2020 and 10th June, 2020 in our tertiary care teaching institute at Hyderabad. The selfdesigned questionnaire consisted of socio-demographic information such as age, gender and education status, and 14 questions based on knowledge and perception towards the disease and its preventive aspects. The questionnaire was validated by external review by the hospital COVID task force committee after pilot testing. Questions about preventive measures were based on the standard operating procedure on preventive measures released by Ministry of health and family welfare (MOHFW), Government of India (6) and CDC guidelines on "What to do when sick?" (7). Attendants of the patients coming to the emergency department (ED) of our hospital in the metropolitan area of Hyderabad were approached. Those willing to participate in the survey were explained about the questionnaire and were asked to choose the appropriate answers to the questions. The questionnaire was primarily prepared in English and Telugu languages and the attendants responded by choosing the appropriate answers for the questions. Illiterates were assisted by explaining the questions in the language they understood and the responses were recorded.

Assuming that more than $50 \%$ of the attendants will be answering more than 7 questions and error of 5\%, the sample size was calculated to be 400 . More than 700 attendants were approached and out of them 509 responded to the questionnaire, with the response rate of around $72 \%$. Data entry and statistical analysis were performed with statistical package for social science software program for Windows (version 18.0). Descriptive statistics, such as percentages, mean and standard deviations were calculated. Independent $t$ test and oneway ANOVA test were used to compare knowledge with demographic characteristics and $P$ value less than 0.05 was considered as statistically significant.

\section{Results}

A total of 509 attendants responded to the questionnaire and $72.9 \%$ of them were males and $27.1 \%$ were females. Among them, $48.1 \%$ belonged to the age group of 30 to 49 years, $44.4 \%$ to 16 to 29 years and $7.5 \%$ were aged more than 50 years. When education was considered, majority of the population had a graduation degree and above (42.4\%). $28.1 \%$ were illiterates and the rest of them were in the categories of primary education (7.3\%), high school $(12.8 \%)$ and Intermediate/ diploma (9.4\%) (Table 1).

It was observed that mass media like newspapers and television had a significant role where the majority of participants heard about COVID-19 (58.0\%). It was followed by social media (20.2\%) and their workplace (11.0\%). Unfortunately, 17 participants (3.3\%) did not hear about COVID-19 (Figure 1).

The overall awareness of COVID-19 among the patient attendants was less than satisfactory. Participants had good knowledge about the origin of the disease, with $87.6 \%$ answering correctly and also the preventive measures of the disease with $72.7 \%$ positive responses. Knowledge about the incubation period, spread of the disease and the immediate measures to be taken if COVID-19 symptoms developed were fairy average with $59.3 \%, 55.6 \%$ and $51.5 \%$ giving correct responses, respectively. The awareness was meager in the aspects of knowledge concerning symptoms of COVID-19 (39.5\% correct responses), asymptomatic transmission $(30.3 \%$ correct responses), period of isolation recommended (21.4\% correct responses), available diagnostic test ( $41.8 \%$ correct responses) and the treatment available (33\% correct responses) (Table 2).

While comparing the demographics with the accuracy of responses, the following was observed. There was no significant difference in the knowledge of males and females and there was no correlation between gender and the knowledge about COVID-19 $(P=0.45)$. It was identified that participants aged between 16 to 29 years had better knowledge than their older counterparts

\section{Where the participants heard about COVID19}

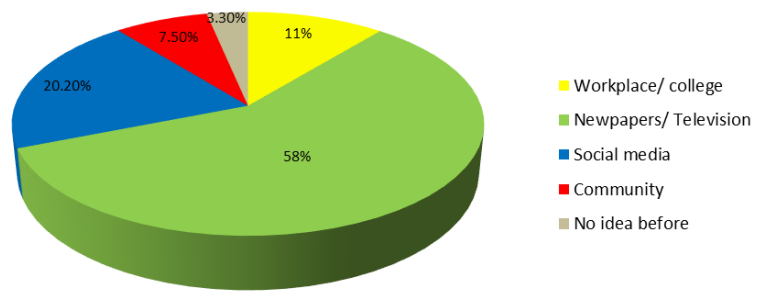

Figure 1. Sources of information regarding the disease.

Table 1. Demographic characteristics of the study sample

\begin{tabular}{lcc}
\hline Demographic & Number & Percent \\
\hline Gender & 371 & 72.9 \\
Male & 138 & 27.1 \\
Female & & \\
Age & 226 & 44.4 \\
16-29 & 245 & 48.1 \\
$30-49$ & 38 & 7.5 \\
$\geq 50$ & & \\
Education & 143 & 28.1 \\
Illiterate & 37 & 7.3 \\
Primary & 65 & 12.8 \\
High school & 48 & 9.4 \\
Intermediate or diploma & 216 & 42.4 \\
Graduate or postgraduate & & \\
\hline
\end{tabular}


Table 2. Responses to questionnaire

\begin{tabular}{|c|c|c|c|c|}
\hline \multirow{2}{*}{ Statements } & \multicolumn{2}{|c|}{ Correct } & \multicolumn{2}{|c|}{ Incorrect } \\
\hline & No. & $\%$ & No. & $\%$ \\
\hline What is COVID-19? & 219 & 43 & 290 & 57 \\
\hline Where did COVID-19 originate? & 446 & 87.6 & 63 & 12.4 \\
\hline What are the symptoms of COVID-19? & 201 & 39.5 & 308 & 60.5 \\
\hline Which of the symptoms are unlikely to be of COVID $19 ?$ & 65 & 12.8 & 444 & 87.2 \\
\hline Can COVID-19 be caught from a person who is not having any symptoms? & 154 & 30.3 & 355 & 69.7 \\
\hline How long does it take for the symptoms of to appear after contracting the disease? & 302 & 59.3 & 207 & 40.7 \\
\hline $\begin{array}{l}\text { How long A person with COVID-19 requires home isolation after being discharged } \\
\text { from hospital? }\end{array}$ & 109 & 21.4 & 400 & 78.6 \\
\hline How does Corona virus spread? & 283 & 55.6 & 226 & 44.4 \\
\hline Do you know any specific test for COVID-19 diagnosis? & 213 & 41.8 & 296 & 58.2 \\
\hline Do you know any treatment for COVID-19? & 168 & 33 & 341 & 67 \\
\hline Do you know the percentage of death in a person infected with COVID 19 in India? & 131 & 25.7 & 378 & 74.3 \\
\hline Your immediate response to COVID-19 symptoms? & 262 & 51.5 & 247 & 48.5 \\
\hline $\begin{array}{l}\text { Which mask is recommended for general public to prevent the spread of COVID } \\
19 \text { ? }\end{array}$ & 200 & 39.3 & 309 & 60.7 \\
\hline Do you know how to prevent the spread of COVID $19 ?$ & 370 & 72.7 & 139 & 27.3 \\
\hline
\end{tabular}

$(P=0.0001)$. Also, it was found that education level corresponded positively with the level of knowledge with participants having a graduate degree or above giving more correct responses $(P=0.0001)$ (Table 3$)$.

\section{Discussion}

To the best of our knowledge, the present study is the first one to assess the awareness of COVID-19 among patient attendants in the city of Hyderabad. The study design included a wide range of age groups categorized into 16 to 29 years, 30 to 50 years and above 50 years of age. Despite many educational programs available, a majority of the participants were not aware about the symptoms of COVID-19, its transmission and the rules for isolation and quarantine. The knowledge about prevention of the spread is quite satisfactory though. These attendants could be potential carriers of the disease and lack of awareness among them carries a greater risk of transmission. Educating them about the common symptoms can give them insight to seek early care both for them and their patients.

While the incubation period of COVID-19 ranges from two days to fourteen days, the average incubation period is five days (8). Majority of the participants knew about the incubation period.

The most common clinical feature was fever, occurring in about $88 \%$ of the cases followed by cough (68\%). Other symptoms included fatigue (38\%), shortness of breath (18\%), myalgias and loss of smell. Diarrhea and vomiting were reported in about 4 and $5 \%$ of the cases, respectively (9). Participants' knowledge regarding the common symptoms was poor and this could prevent them from seeking timely care.

Many of the prevention strategies are advised by Centre for Disease Control and Prevention (CDC) and the WHO. The MOHFW in India has also released guidelines for the prevention of COVID-19 in the following aspects, viz., $(i)$
Generic preventive measures to be followed at all times, (ii) Measures specific to offices, (iii) Measures to be taken on occurrence of case(s), (iv) Disinfection procedures to be implemented in case of occurrence of suspect/ confirmed cases (6).

General prevention strategies include:

- MOHFW recommends maintaining at least six feet of distance between persons as a part of physical distancing strategy (6).

- The CDC recommends washing hands with soap and water for at least twenty seconds or using alcohol (60\% solutions) based hand rubs when soap and water are not available (7).

- WHO, in June 2020, advised wearing masks in public (10).

- Social distancing is another effective measure intended to slow down the disease transmission by minimizing the close contact with individuals. Methods include quarantine, travel restrictions, closure of areas of social gatherings, etc.

There is a concept of "Flattening the curve", where the

Table 3. Demographics and correlation with correctness of responses

\begin{tabular}{lccc}
\hline Demographic & MEAN \pm SD & t/F value & $P$ value \\
\hline Gender & & & \\
$\quad$ Male & $6.09 \pm 2.3$ & & \\
$\quad$ Female & $6.26 \pm 2.3$ & 0.751 & 0.45 \\
Age & & & \\
16- 29 & $6.83 \pm 2.0$ & & \\
30- 49 & $5.66 \pm 2.4$ & 21.73 & 0.0001 \\
50 and above & $5.00 \pm 1.73$ & & \\
Education & & & \\
Illiterate & $5.3 \pm 2.1$ & & \\
Primary & $4.3 \pm 2.3$ & & \\
High school & $5.8 \pm 2.0$ & 23.09 & \\
Intermediate or diploma & $6.2 \pm 2.3$ & & \\
Graduate or post graduate & $7.1 \pm 2.1$ & & \\
\hline
\end{tabular}


preventive measures are enforced to delay the epidemic peak. This helps to reduce the infection rate to avoid health services being overwhelmed and to allow better care for current cases and delay the occurrence of additional cases until effective treatment or vaccine is available (11). The participants were mostly aware of the preventive strategies like social distancing and personal hygiene. Government of India has taken initiatives like giving advisories over calls to spread the awareness of preventive aspects.

Coming to the treatment aspects, all the anti-viral medications are under investigation and none has yet been shown to be clearly effective on mortality. Antivirals like remdesivir, favipiravir, lopinavir/ritonavir have been used with some benefits. Anti-malarial, Hydroxychloroquine was extensively used. But in June, use of hydroxychloroquine in the UK "Recovery trial" was discontinued when an interim analysis of 1542 treatments showed that it had no mortality benefit to people hospitalized with severe COVID-19 infection over 28 days of observation (12). Apart from specific drugs, the treatment also includes supportive measures like various oxygenation and ventilation strategies. The participants also lacked proper awareness of the treatment options available to them.

Bearing the above in mind, the need for educating the patient attendants will have a pivotal role in curbing the spread of the disease as these are the population that acts as a link between the hospitals and the general public.

Our survey showed that the knowledge and awareness regarding the disease is equal between genders but findings showed a positive correlation with the level of education. Age groups between 16 and 29 years were more aware of the disease followed by adults between 30 and 50 years.

\section{Conclusion}

Our survey showed that the awareness of the patient attendants was less than satisfactory. This shows the need for more educational programs and awareness campaigns. Also, it indicates the need for participation of health care workers in not just attending the patients but also to put an effort to educate the attendants. As mentioned earlier, the attendants act as a link between the hospital and the general public. This, apart from being a potential route for the disease to spread, also gives the opportunity to exploit the link to indirectly educate the masses. It is unfortunate that knowledge of preventive measures is not fully getting translated into strict compliance of the guidelines due to lack of discipline, a sort of callousness which is evident in not wearing masks, overcrowding, not maintaining the social distance even by the educated as well.

With the hopes of vaccine coming out in near future appearing bleak, it is still essential to rely on preventive measures to flatten the curve of the pandemic. To achieve this, there is a need for combined efforts of government institutes and mainly hospitals to enlighten the individuals to reduce fear and false beliefs and promote preventive strategies.

By and large, public are aware that a deadly pandemic is staring at them without a medicine to treat it. So the need of the hour is to sensitize the illiterate public, through various media for strict compliance of the COVID-19 guidelines in addition to impressing the informed but violating public for strict compliance of guidelines. These steps are necessary for attendants of the patients who carry more risk of contracting and spreading of the COVID-19.

\section{Authors' contributions}

Study design, MIN; study conduct, MMR; data gathering, GDR and USC; supervision, AS; data analysis and writing, LT and MMR; critic, AS and MIN.

\section{Ethical issues}

Nizam's Institute of Medical Sciences approved the study (Approval ID PBAC No. 1255/2020).

\section{References}

1. Hui DS, Azhar EI, Madani TA, Ntoumi F, Kock R, Dar O, et al. The continuing 2019-nCoV epidemic threat of novel coronaviruses to global health - The latest 2019 novel coronavirus outbreak in Wuhan, China. Int J Infect Dis 2020; 91: 264-6. doi: 10.1016/j.ijid.2020.01.009.

2. Cucinotta D, Vanelli M. WHO declares COVID-19 a pandemic. Acta Biomed 2020; 91(1): 157-60. doi: 10.23750/ abm.v91i1.9397.

3. Johns Hopkins University. Coronavirus COVID-19 Global Cases by Johns Hopkins CSSE. Johns Hopkins University; 2020. Available from: https:/gisanddata. maps.arcgis.com/apps/opsdashboard/index.html\#/ bda7594740fd40299423467b48e9ecf6.

4. Sarraf DP, Gupta PP, Keshwar S. Public's knowledge and beliefs towards universal safety precautions during COVID-19 pandemic in Nepal: a web-based cross-sectional study. J Drug Deliv Ther 2020; 10(3 Suppl): 133-41. doi: 10.22270/jddt.v10i3-s.4175.

5. Zhong BL, Luo W, Li HM, Zhang QQ, Liu XG, Li WT, et al. Knowledge, attitudes, and practices towards COVID-19 among Chinese residents during the rapid rise period of the COVID-19 outbreak: a quick online cross-sectional survey. Int J Biol Sci 2020; 16(10): 1745-52. doi: 10.7150/ijbs.45221.

6. Ministry of Health and Family Welfare, Government of India. SOP on Preventive Measures to Contain Spread of COVID-19 in Offices. Ministry of Health and Family Welfare, Government of India; 2020. Available from: https:// www.mohfw.gov.in/pdf/1SoPstobefollowedinOffices.pdf.

7. Centrefor Disease control and prevention-COVID 19-steps to do when you are sick. Available from: https://www.cdc. gov/coronavirus/2019-ncov/if-you-are-sick/steps-whensick.html?CDC_AA_refVal=https\%3A\%2F\%2Fwww. cdc.gov\%2Fcoronavirus\%2F2019-ncov\%2Fif-you-aresick\%2Fcaring-for-yourself-at-home.html.

8. Lauer SA, Grantz KH, Bi Q, Jones FK, Zheng Q, Meredith $\mathrm{HR}$, et al. The incubation period of coronavirus disease 2019 (COVID-19) from publicly reported confirmed cases: estimation and application. Ann Intern Med 2020; 172(9): 577-82. doi: 10.7326/m20-0504. 
9. Tu H, Tu S, Gao S, Shao A, Sheng J. Current epidemiological and clinical features of COVID-19; a global perspective from China. J Infect 2020; 81(1): 1-9. doi: 10.1016/j. jinf.2020.04.011.

10. BBC News. Coronavirus: WHO Advises to Wear Masks in Public Areas. BBC News; 2020. Available from: https:// www.bbc.com/news/health-52945210.

11. Anderson RM, Heesterbeek H, Klinkenberg D, Hollingsworth TD. How will country-based mitigation measures influence the course of the COVID-19 epidemic?
Lancet 2020; 395(10228): 931-4. doi: 10.1016/s01406736(20)30567-5.

12. Recovery trial - statement from chief investigators on use of hydroxychloroquine in hospitalized patients with COVID 19. Available from: https://www.recoverytrial. net/news/statement-from-the-chief-investigators-of-therandomised-evaluation-of-covid-19-therapy-recoverytrial-on-hydroxychloroquine-5-june-2020-no-clinicalbenefit-from-use-of-hydroxychloroquine-in-hospitalisedpatients-with-covid-19. 